\title{
A Novel Idea for Optimizing Condition-Based Maintenance Using Genetic Algorithms and Continuous Event Simulation Techniques
}

\author{
Mansoor Ahmed Siddiqui, ${ }^{1}$ Shahid Ikramullah Butt, ${ }^{1}$ Aamer Ahmed Baqai, ${ }^{2}$ \\ Jiping Lu, ${ }^{3}$ and Faping Zhang ${ }^{3}$ \\ ${ }^{1}$ Department of Design \& Manufacturing Engineering, SMME, NUST H-12 Campus, Islamabad 44000, Pakistan \\ ${ }^{2}$ Department of Mechanical Engineering, CEME, NUST, Rawalpindi 46000, Pakistan \\ ${ }^{3}$ School of Mechanical Engineering, Beijing Institute of Technology, Beijing 100081, China \\ Correspondence should be addressed to Mansoor Ahmed Siddiqui; mani_290@yahoo.com
}

Received 27 October 2016; Revised 28 December 2016; Accepted 4 January 2017; Published 31 January 2017

Academic Editor: Yaguo Lei

Copyright (C) 2017 Mansoor Ahmed Siddiqui et al. This is an open access article distributed under the Creative Commons Attribution License, which permits unrestricted use, distribution, and reproduction in any medium, provided the original work is properly cited.

\begin{abstract}
Effective maintenance strategies are of utmost significance for system engineering due to their direct linkage with financial aspects and safety of the plants' operation. At a point where the state of a system, for instance, level of its deterioration, can be constantly observed, a strategy based on condition-based maintenance (CBM) may be affected; wherein upkeep of the system is done progressively on the premise of monitored state of the system. In this article, a multicomponent framework is considered that is continuously kept under observation. In order to decide an optimal deterioration stage for the said system, Genetic Algorithm (GA) technique has been utilized that figures out when its preventive maintenance should be carried out. The system is configured into a multiobjective problem that is aimed at optimizing the two desired objectives, namely, profitability and accessibility. For the sake of reality, a prognostic model portraying the advancements of deteriorating system has been employed that will be based on utilization of continuous event simulation techniques. In this regard, Monte Carlo (MC) simulation has been shortlisted as it can take into account a wide range of probable options that can help in reducing uncertainty. The inherent benefits proffered by the said simulation technique are fully utilized to display various elements of a deteriorating system working under stressed environment. The proposed synergic model (GA and MC) is considered to be more effective due to the employment of "drop-by-drop approach" that permits successful drive of the related search process with regard to the best optimal solutions.
\end{abstract}

\section{Introduction and Literature Review}

Optimization of maintenance activity is considered to be an interesting subject for scientists and researchers, as can be observed by the abundantly available related conference papers and journals, and also to the industry, for its notable fallbacks to the activities associated with safety and financial aspects.

Distinctive ways and approaches are available to deal with the issue of maintenance. Generally, maintenance is carried out on a restorative premise or planned occasionally based on experience of the professionals. When state can be observed, constantly for the functional systems through testing or investigations for the reserved safety frameworks, a CBM policy can be enforced, which helps in deciding when the maintenance of system should be carried out. Benefits of CBM strategy exist in the likelihood of maintaining the framework just when required, thus, sparing assets as well as the system's accessibility at the very basic level. This strategy indicates remarkable possibilities in frameworks like atomic power plants, costal establishments, and aviation setups, which work under severe conditions which can harm their coherence and usefulness while being consistently observed due to the safety repercussions.

During design stage, the need of building up a productive CBM policy is twofold. Firstly, one needs to build up an 
appropriate perceptive model for the system, depicting its future development of deterioration level in the light of observed factors; secondly, one must have the capacity to assess diverse maintenance procedures in an optimization plan aimed at optimizing two main objectives, namely, profitability and accessibility.

Accessibility is a measure of predefined operable and committed state of a framework, its subsystems, or components at the beginning of an operation, when the time of operation is quite obscure, that is, arbitrary or random. In other words, accessibility is the extent of time a framework is in a working condition. It is also referred to as "capable rate" of an operation. Numerically, it is equal to $100 \%$ minus the time when a system is inaccessible.

Numerous researchers and scientists have concentrated on the issue of developing thorough models for degrading systems. In this regard, Markov's chain and Markov's model have been mostly used for accomplishing the logical outcomes [1-7]. However, in the abovementioned cases, models are generally based on simple hypotheses. Most of the models available in literature presume that the level of system's deterioration can be ascertained by means of repeated examination only [2, 4, 5, 7]; Kopnov [6] considered a situation where the system is consistently observed. Lately, Lam and Yeh [3] considered both cases. An additional hypothesis is to believe that repairs/replacement of the spare parts always reestablish the system to a "perfect" condition, which may not be exceptionally practical. As of late, Kopnov [6] has considered an issue of limited recuperation. Whenever more practical issues related to dynamic conduct of the system should be taken into account, simple investigative models cannot realistically depict the system, which ultimately forced the researchers to adopt the simulation techniques like the Monte Carlo simulation [5, 7-9].

As far as optimization of maintenance system is concerned, the issue fundamentally sums up to decide the level of the observed deteriorated condition of the system after which maintenance should be carried out. Traditional optimization techniques, for example nonlinear programming, dynamic programming, gradient methods, mixed integer, and integer programming, regularly involve the utilization of improved models, giving clear assertions regarding optimization of cost functions and, perhaps, their derivatives. As already emphasized, the unpredictable performance of the contemporary manufacturing plants and other industrial setups can barely be managed using simpler optimization models as it is likely that the objective functions and their dependent variables are fixed into complex and more complicated computer codes. Thus, a serious impediment exists related to the abovementioned optimization methods in today's dynamic environment.

The researchers are confronted with the daunting challenge of accomplishing several objectives at the same time while trying to optimize a design feature of any engineered framework, such as higher revenues, high dependability, low costs, and low risk of accidents, which may offset each other. Moreover, a few strict requirements pertaining to weight/volume of the system are also required to be fulfilled.
In order to optimize a multiobjective system, the optimization search methods focused on an individual objective function, being a factored merger of desired objectives while inflicting necessary constraints on the system at the same time, are generally used [10-13]. This methodology certainly leads to unpredictable definition of the levels of constraints and weighted factors that have been used to optimize the system.

A more useful method is to consider every single objective independently and to identify a set of solutions that is relatively better. Every element of this set is considered to be better or equivalent to the other elements of the set, barring few odds, as far as desired objectives are concerned. Therefore, a set of solutions obtained as a result of multiobjective methodology, described above, gives a range of "satisfactory" solutions between which trade-off can be reached.

In our research, a methodology has been proposed that couples the Genetic Algorithms (GAs) [14-29] with a continuous event simulation technique so as to display a more sensible prognostic model portraying the deteriorating system and thereby seeking an optimal deterioration level after which preventive maintenance must be carried out while optimizing various objectives at the same time. In this regard, Monte Carlo (MC) simulation technique has been shortlisted as it takes into account a wide range of probable options that can help in reducing uncertainty [11, 30-33]. Basically, we consider "mean accessibility" and "total profit," which resulted from the operating system over a specified time, as our "main objectives." A more common potential solution consists of encoded chromosomes of Genetic Algorithms (GA) population, comprising a set of variables, one of each type, so as to decide the optimal deterioration level after which preventive maintenance is necessitated. All the potential solutions obtained as a result of GA search will be evaluated using proposed model of MC simulation in order to accomplish the objectives of profitability and accessibility. This coupled approach has already been used by few researchers for both single $[12,31]$ as well as multiobjective [33] issues. Although different algorithms for Multiple Objective Genetic Algorithm (MOGA) and MC simulation are available, however, for the sake of simplicity and clarity, in-built algorithms offered by MATLAB have been utilized for carrying out the requisite calculations.

The paper is organized in different sections. Section 2 describes problem statement, whereas in Section 3 Markov's model for deteriorating system has been defined. Keeping in view various probabilities, effects of maintenance on a particular component in the form of either increased deterioration level or improved condition of the same are highlighted in the said model, in case a maintenance activity is performed or otherwise. Moreover, Monte Carlo (MC) simulation model of the system is also described in the same section. In Section 4, the basics of GA search methodology are briefly presented, while encompassing the subject of multiobjective optimization in further details. The reasons for using this coupled approach (i.e., GA and MC) and the proposed model are stated in Section 5 along with its Pseudocode 1. Simulated results for the proposed model 


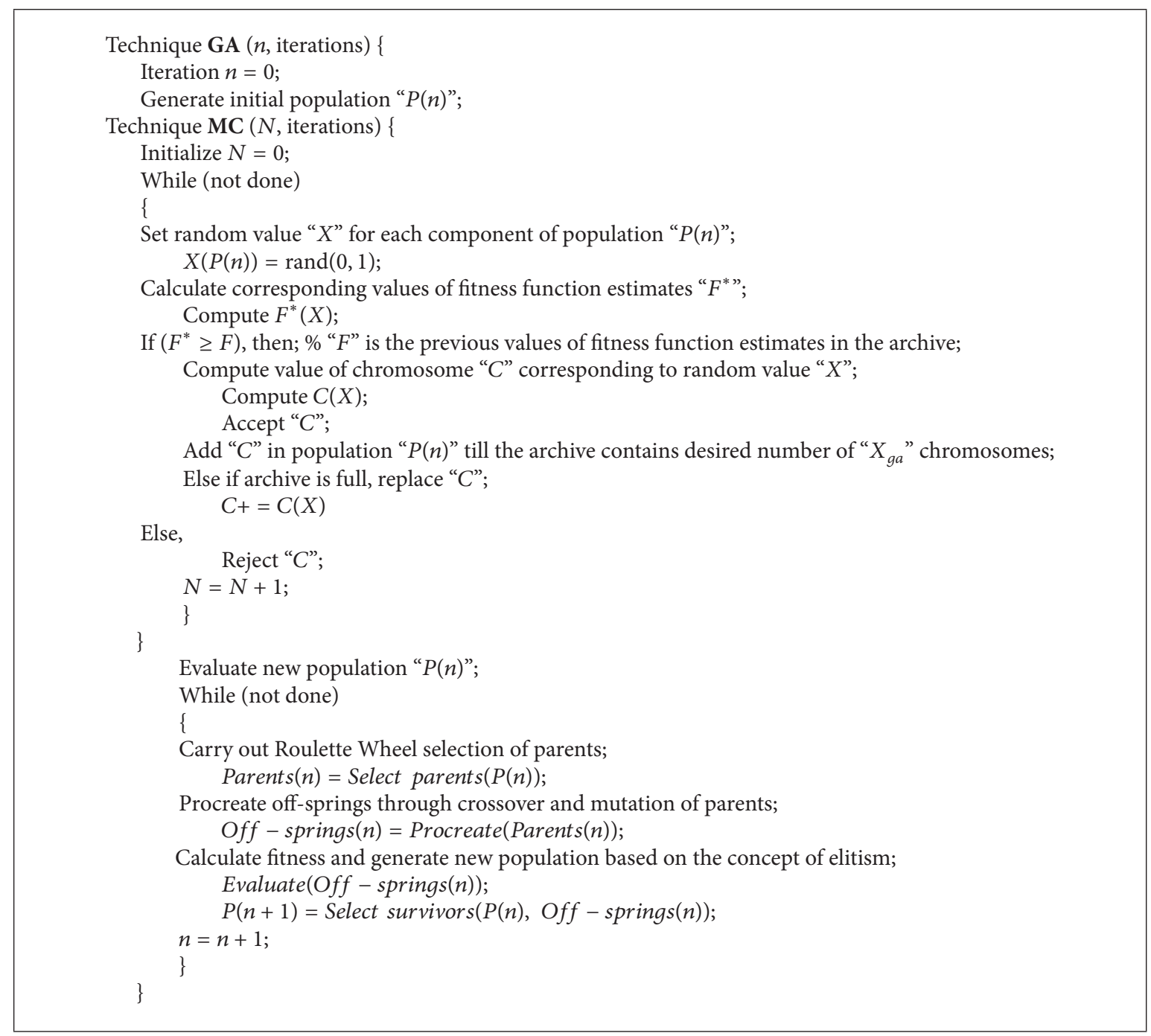

Pseudocode 1

are presented in Section 6 and conclusion is finally made in Section 7 of the article.

\section{Problem Formulation}

Let us review a framework comprising " $N_{n}$ " nodes (macrocomponents) in series, each accomplishing its specific task (Figure 1). Every " $i_{n}$ th" node consists of " $N_{p}\left(i_{n}\right)$ " parallel branches, wherein each branch of the node contains a series of " $N_{s}\left(i_{n}, i_{p}\right)$ " components, $i_{n}=1,2, \ldots, N_{n}$ and $i_{p}=$ $1,2, \ldots, N_{p}\left(i_{n}\right)$. If " $N_{t}$ " types of components are present in the system, then total components in the system can be given by following expression:

$$
N_{c}=\sum_{i_{n}=1}^{N_{n}} \sum_{i_{p}=1}^{N_{p}\left(i_{n}\right)} N_{s}\left(i_{n}, i_{p}\right) .
$$

It is assumed that same maintenance policy will be adopted for the components of similar type and the performance of components will deteriorate with the passage of time. Other assumptions made for the system are as follows:

(i) The level of degradation for each component is continuously updated.

(ii) Each component has its probability of failure as a function of its deterioration level.

(iii) Each component is subjected to CBM, where the deterioration falls beyond a given level.

The issue in this regard is to determine an optimal deterioration level beyond which the maintenance is necessitated. The issue is configured as an optimization search based on multiple objective, with the aim to maximize the profitability, " $G$," and the mean accessibility, " $\bar{A}$," of the system during a given time " $T_{M}$." The mean accessibility, " $\bar{A}$," of the system with regard to the deteriorating components will be discussed further in Section 3. As far as the profitability, " $G$," is concerned, it can be expressed by following equation:

$$
G=P_{t}-(M+C)
$$




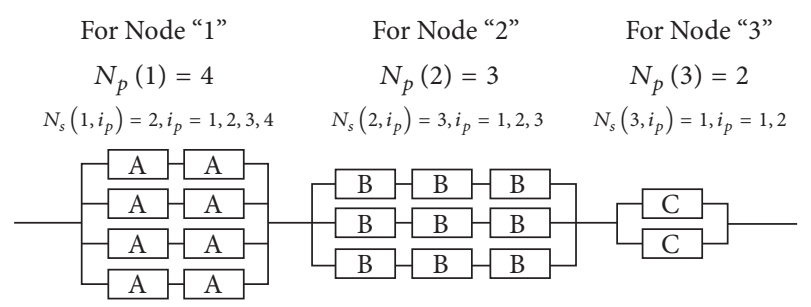

Figure 1: A framework of $N_{n}=3$ nodes, comprising $N_{t}=3$ component types (A, B, and C).

where $P_{t}=S \int_{0}^{T_{M}} A(t) d t$ is the overall profit of plant, " $A(t)$ " is the accessibility of plant at time " $t$," and " $S$ " are the service charges for service of the plant paid by customer per unit time.

$M=\sum_{i=1}^{N_{c}} M_{i} \int_{0}^{T_{M}} P_{M}^{i}(t) d t$ are the aggregate maintenance charges for all elements of the system, $i=1, \ldots, N_{c}$; " $M_{i}$ " are the maintenance charges for an element " $i$ " and " $P_{M}^{i}(t)$ " is the probability of " $i$ th" element being maintained at a time " $t$."

$C=C_{U} \int_{0}^{T_{M}} \mathrm{ND}(t) d t$ is the compensation paid to the customer due to nondelivery " $\mathrm{ND}(t)$ " of consented services when the system is nonfunctional; " $C_{U}$ " is the monetary penalty imposed and $\mathrm{ND}(t)=1-A(t)$, with " $A(t)$ " being the accessibility of plant at time " $t$."

In order to evaluate system's performance, different mathematical and analytical methods exist. For better comprehension and drawing necessary conclusion with regard to their suitability and effectiveness, few of these will be considered in the following sections.

\section{Markov's Model and MC Simulation for Deteriorating System}

3.1. Markov's Model. Markov's process is a numerical method that transforms a system from one state to another, within a limited number of feasible states. Markov was of the view that future state of the system is not dependent on its past states when its present state is clearly established.

In order to understand Markov's Model correctly, we need to first get clarity about the Markov's Chain. If $Y_{x+1}=m$ shows a stochastic/probabilistic procedure that is supposed to remain at a state " $m$ " for the time " $x+1$ ", then

$$
\begin{aligned}
& P\left(Y_{x+1}=m \mid Y_{x}=n, Y_{x-1}=n_{x}, \ldots, Y_{2}=n_{2}, Y_{1}\right. \\
& \left.\quad=n_{1}, Y_{0}=n_{0}\right) \\
& P\left(Y_{x+1}=m \mid Y_{x}=n\right)=P_{n m} .
\end{aligned}
$$

for the states $m, n, n_{x}, \ldots, n_{0}$ and all $x \geq 0$. This kind of probabilistic procedure is termed as Markov's chain. The value " $P_{n m}$ " indicates the probability of stochastic procedure to transform the system from state " $n$ " to " $m$." As per Ross's theory, all the probabilities are greater than or equal to " 0 " and that the stochastic procedure must transform to the other states; thus

$$
\begin{aligned}
P_{n m} & \geq 0, \\
\sum_{m=0}^{\infty} P_{n m} & =1, \quad n=0,1,2, \ldots .
\end{aligned}
$$

Representing the " $x+y$ " probabilities of system transition by $P\left(X_{x+y}=m \mid Y_{0}=n\right)=P_{n m}^{x+y}$ and computing the same by Chapman-Kolmogorov's equation, we get

$$
P_{n m}^{x+y}=\sum_{k=0}^{\infty} P_{n k}^{x} P_{k m}^{y}
$$

for all $x, y \geq 0$. In (5), the term " $P_{n k}^{x} P_{k m}^{y}$ " shows probability of system's transformation from state " $n$ " to " $m$ " during " $x+y$ " transitions following a path that will take the system to state " $k$ " after " $x$ th" transition.

In general, Markov's process can be termed as an adjunct to the Markov's chain. If $E=\{0,1,2,3, \ldots\}$ denotes the state space and $(Y(t) \mid t \geq 0)$ represents a Markov's process, then as per Markov's chain definition, $(Y(t) \mid t \geq 0)$ is called a "continuous-time Markov's chain," wherein states $n_{x}, n, m \in E$ and $0 \leq z_{1}<z_{2}<\cdots<z_{x}<t^{*}<t^{*}+t$. Mathematically, the same can be expressed as follows:

$$
\begin{aligned}
& P_{n m}\left(t^{*}, t^{*}+t\right)=P\left(Y\left(t^{*}+t\right)=m \mid Y\left(t^{*}\right)=n, Y\left(z_{x}\right)\right. \\
& \quad=n_{x}, Y\left(z_{x-1}\right)=n_{x-1}, Y\left(z_{x-2}\right)=n_{x-2} \cdots Y\left(z_{1}\right) \\
& \left.=n_{1}\right)=P\left(Y\left(t^{*}+t\right)=m \mid Y\left(t^{*}\right)=n\right),
\end{aligned}
$$

where " $P_{n m}\left(t^{*}, t^{*}+t\right)$ " is the transition probability which indicates that if a system were at state " $n$ " at time " $t$," then it will move to the next state " $m$ " at time " $t$ * $+t$."

Markov's process is considered to have Markovian property which affirms that the future state of a system will be independent of its past state " $Y\left(z_{k}\right)=n_{k}$," provided present state " $Y\left(t^{*}\right)=n$ " of the system is known. Therefore, (6) can be elucidated as follows:

$$
P_{n m}(t)=P\left(Y\left(t^{*}+t\right)=m \mid Y\left(t^{*}\right)=n\right) .
$$

The abovementioned Markov process is known as "continuous-time homogeneous Markov's process," where " $P_{n m}(t)$ " is the probability of transition of Markov's process that depends on the length of time interval " $t$ " rather than the actual time " $t$ "."

In case (6) cannot be elucidated as (7), it shows that the transition probability depends on the actual time interval " $\left(t^{*}, t^{*}+t\right)$ " as well as the starting time " $t$." As per the definitions of stationary and homogeneous transition probabilities [34], these are always modified with respect to time, which is termed as "continuous-time inhomogeneous Markov process."

Chapman-Kolmogorov equation of "continuous Markov process" is, therefore, the solution of transition probabilities within a particular time interval. The said equation is as follows:

$$
\begin{aligned}
P_{n m}\left(t^{*}+t\right) & =P\left(Y\left(t^{*}+t\right)=m \mid Y(0)=n\right) \\
& =\sum_{k \in E} P_{n k}\left(t^{*}\right) P_{k m}(t)
\end{aligned}
$$




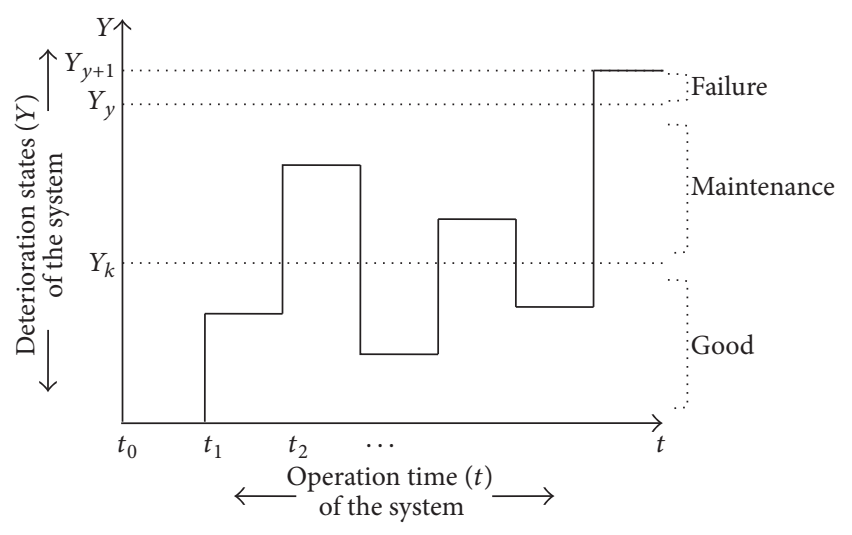

Figure 2: Deterioration process of an element in a given time.

for $n, m, k \in E$. Equation (8) illustrates that the system will move to condition " $m$ " during a time period " $t$ * $+t$ " starting from condition " $n$ " on a path that would take the system towards condition " $k$ " at time " $t$ "." A feasible interpretation of the time progression of a component is depicted in Figure 2.

Finally, the system's accessibility " $A(t)$ " at a given time " $t$ " along with its probability of being under repair " $P_{M}(t)$ " can be expressed using the following equations:

$$
\begin{aligned}
A(t) & =\sum_{n=0}^{k} \sum_{m=1}^{k+1} P_{n m} \\
P_{M}(t) & =\sum_{n=k+1}^{y} \sum_{m=k+2}^{y+1} P_{n m} .
\end{aligned}
$$

Using the abovementioned Markov Model, objective function as well as the mean system accessibility " $A(t)$ " over a given time " $t$ " can be computed. However, a systematic approach to evaluate the system accessibility is possible only if simplified hypotheses are made. Therefore, a more pragmatic approach towards modeling of the process is essentially required. In order to deal with such situations, Monte Carlo (MC) simulation provides a better bailout solution.

3.2. MC Simulation. In the field of reliability sciences, simulation can be termed as a good substitute to the analytical techniques. Prediction of component's accessibility within a given timeframe by MC simulation is the implementation of the said concept. It is pertinent to highlight that the simulation results acquired by $\mathrm{MC}$ simulation conform to the ones achieved with Markov's process that is considered to be a more traditional technique.

MC simulation can be described in many ways; however, one thing that is common in all the definitions is that it carries out random sampling to reach a solution. The algorithms of MC simulation depend on "pseudorandom numbers" to generate a feasible outcome of the process. All realizations are expected to have different probabilities, and by repeating the process using different "pseudorandom numbers" as input, accurate data regarding modeled processes is obtained. Based on the acquired data, a statistical analysis can then be performed so as to answer various questions with regard to the process.

After several MC simulation runs, we achieve numerous independent interpretations of the abovementioned pseudorandom variables whose group averages provide estimates of the system accessibility " $A(t)$ " as well as the probability of component " $i$ " being under maintenance " $P_{M}(t)$," where $i=1, \ldots, N_{c}$. Using the said data, an estimate of the desired objective functions, that is, mean accessibility " $\bar{A}$ " and net profit " $G$ " of the system, can be estimated accordingly.

In order to model the dynamics of maintenance, the process strictly depends on the quantity of available maintenance professionals. If none of the professionals is available, due to their commitment on some other components, the faulty equipment has to be kept on hold before its repair can be commenced; that is, in MC simulation, a stochastic transition of a component is allowed towards an operational state only when the quantity of components being repaired simultaneously is less than the quantity of unoccupied maintenance professionals.

\section{Optimizing the Solution through GA}

Genetic Algorithm (GA) was first validated as an optimization technique by Holland in 1975 [15]. It is a search method evolved to imitate the processes of natural evolution. GA is different from other optimization methods owing to its global searching capability accomplished by a population of solutions as opposed to a single solution. Each of the proposed solutions is depicted by a vector " $Y$," comprising variables that are independent of one another, further coded into chromosomes, consisting of different genes, each representing an element of the said vector. Generally, binary coding technique is utilized for the said purpose.

At the start of search process in GA, a randomized initial population of chromosomes, comprising " $X_{g a}$ " feasible solutions, is generated. Next, the evaluation of the said chromosomes in terms of their fitness is carried out. This generated initial population is further evolved during successive iterations. Evaluation of objective function is carried out each time as a fresh solution " $Y$ " is suggested as a result of optimization process. Consequently, individuals' ranking in the present population is updated, keeping in view the values of their individual fitness. The same is then utilized during selection process, giving the best individuals a greater chance of selection as parents. Moreover, ranking of the individuals is also utilized during substitution process in order to decide that whether the parents or the offspring should carry forward to the next population or otherwise. A genetic algorithm is termed as a "steady-state genetic algorithm" based on abovementioned processes [35].

Multiobjective optimization issue occurs when we have to take into account various objective functions $f_{n}(Y), n=$ $1,2, \ldots$, corresponding to each point " $Y$ " in the complete search space, followed by establishment of a point " $Y^{*}$ " that generates the best possible trade-off between different objective functions. Let us take into account " $N$ " number of distinct objective functions $f_{n}(Y), n=1, \ldots, N$ where " $Y$ " exhibits a vector of variables that are independent of each 


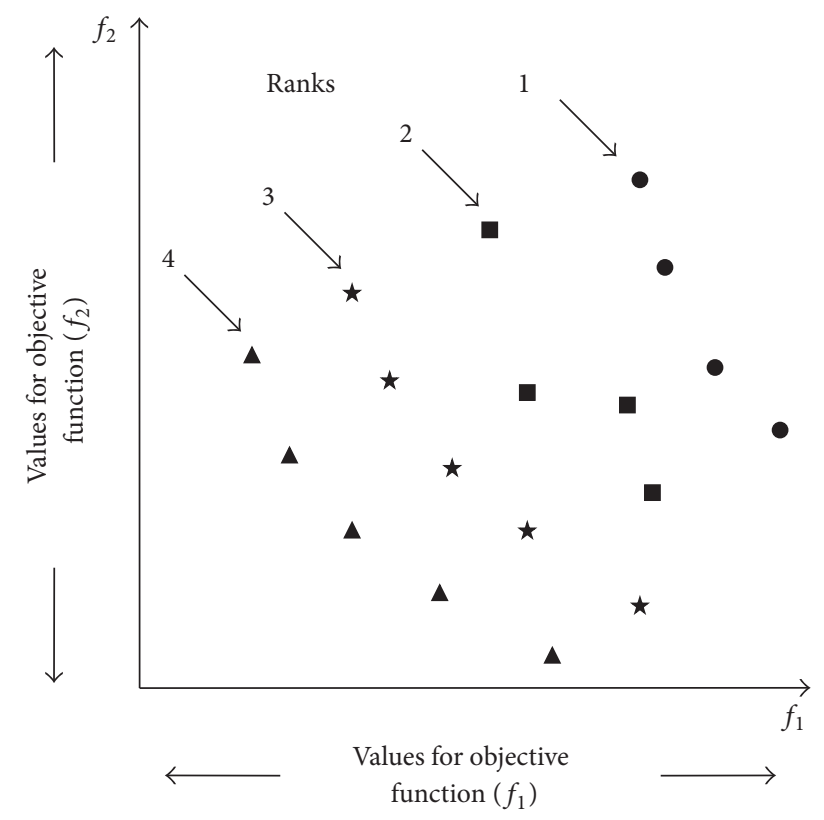

FIGURE 3: Population ranking for maximization of functions $f_{1}$ and $f_{2}$.

other, classifying a proposed generalized solution. Solution " $Z$ " is said to be "dominated" by solution " $Y$," if " $Y$ " displays improved performance for all the objective functions [10], that is, if

$$
f_{n}(Y)>f_{n}(Z) \quad \text { for } n=1,2, \ldots, N
$$

A solution is said to be "nondominated" if none of the cost functions can have improved values without reducing the values of some other objectives. In order to deal with various objective functions simultaneously while following the genetic approach, single-fitness process being used for singleobjective GA problems should be generalized by allocating " $N$ " fitness to solutions " $Y$."

Once the population of chromosomes $[X]$ is created as desired, ranking of the same is carried out as per Pareto domination criteria by going through the $N$-dimensional search space of different fitness $f_{n}(Y), n=1,2, \ldots, N$. Ranking of the chromosomes for $N=2$ is shown in Figure 3. "Nondominated" solutions, in the present population, are determined. The same are then allocated rank "1," being the best ones. After that, these are separated from the other chromosomes. Then again, a fresh set of "nondominated" solutions is selected, which are allocated rank " 2 ." The said procedure continues till the time each individual in the population is ranked.

The selection and substitution process of Multiple Objective Genetic Algorithms (MOGA) depends on the abovementioned ranking. Each chromosome that belongs to the same rank should be regarded as equal to the others of the same group; that is, its probability of selection as a parent and surviving the substitution is equal to any other member of the same group.
While carrying out an optimization search, the information about vectors updated during previous histories, depicting the Pareto efficiency, is noted and upgraded, which consist of "nondominated" chromosomes and their consequent " $N$ " fitness. On completion of each iteration, evaluation of "nondominated" solutions in the present population is carried out with regard to those already recorded, taking their fitness into account. In this regard, the following rules are applied:

(i) If the existing members in the archive are dominated by a new individual, the same are deleted and the new one is made part of the archive.

(ii) If any member of the archive dominates the new individual, it is not added.

(iii) If the new solution is neither dominated nor it dominates any other member of the archive, then

(a) the new individual is added in the archive, in case the same is not full.

(b) in case the archive already has the desired number of solutions, the new individual is exchanged with the one having maximum similarity, present within the record.

Record of "nondominated" solutions is also manipulated by initiating a selection process of elitist parents that is considered to be more effective. Either every individual in the archive is chosen as a parent during different iterations at least once or, in case of a larger archive, the quantity of individuals is earmarked by an already defined ratio of the population " $X_{g a}$ ", generally $25 \%$, that is, $X_{g a} / 4$. This elitism process assures a better implementation of genetic algorithm, resulting into "nondominated" individuals, thus ensuring an effective development of population towards Pareto efficiency, while maintaining the diversity in genetics simultaneously. On completion of the search process, the outcome of optimization consists of an archive that provides the desired Pareto efficiency region.

\section{Proposed Model: MC Embedded in GA}

5.1. Reason for Using the Coupled Approach. The quest for the optimum maintenance levels necessitates a choice to be made amongst a large number of feasible substitutes. Obviously, running a complete MC simulation for each alternate solution using precise data for the sake of completing only a rudimentary search process is quite unfeasible. Alternatively, when the search process for optimized solution is led by GA, a MC simulation is required to be performed for each individual of the population being considered during consecutive iterations, which is also not practicable. A feasible solution in this regard arises from the fact that, in GA approach, better chromosomes appear more number of times during consecutive iterations whereas the others are immediately removed. This leads us to consider a coupled approach, encompassing MC as well as GA and thus ensuring better optimized solution to the problem in much lesser time. 


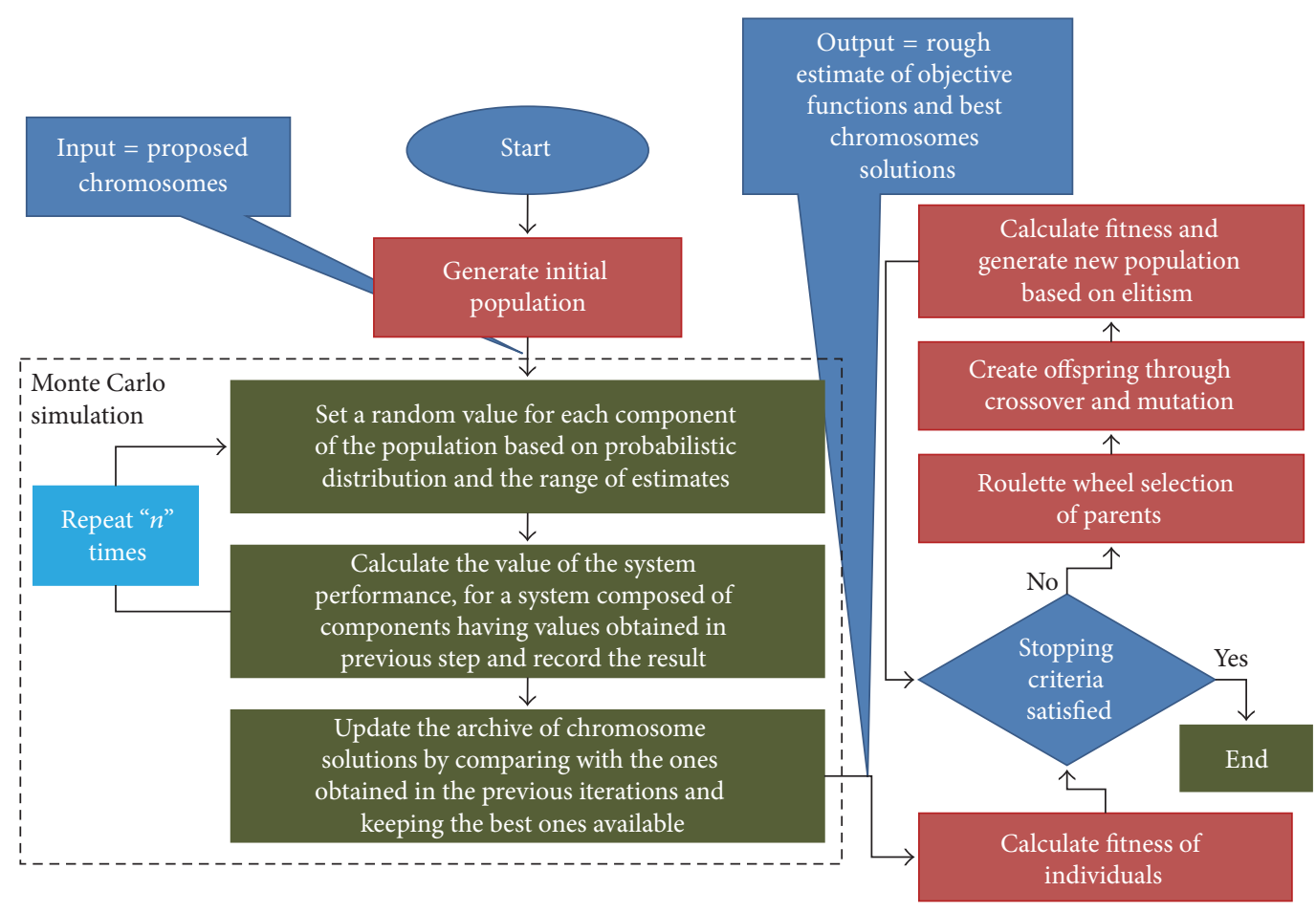

FIgure 4: Proposed model: MC embedded in GA.

5.2. Proposed Model. Based on the abovementioned concept, MC simulation is conducted for each of the suggested chromosomes for a specified number of times, for example, 200 iterations, thus giving a crude approximation of the objective functions. At the time of GA evolution, an update of record of the best chromosome solutions, acquired during preceding $\mathrm{MC}$ iterations, along with their corresponding approximations of the objective functions is carried out. When a chromosome is suggested for the second time, the freshly calculated objective function approximation can be stored with those already available in the archive. This repetition can result in the "best" chromosomes through natural selection procedure while accumulating the outcomes of few last iterations over and over again, thus acquiring arithmetically important results at the end [12]. The said approach is known as "drop-by-drop approach" due to its resemblance with the process of filling a container with liquid. This technique abstains from working on the "bad solutions" that are imitated for less repetitions. The proposed model is illustrated in Figure 4.

5.3. Pseudocode. Pseudocode for the proposed model, embedding MC simulation in GA, is illustrated as under. The same will be utilized for further development of MATLAB code for the suggested approach. Interaction of MC simulation and GA is quite evident in the pseudocode. This interaction is expected to provide better results for CBM optimization problem in less time as compared to other combinatorial algorithms, as shown in Pseudocode 1.
TABLE 1: Arbitrary data of the described framework.

\begin{tabular}{llc}
\hline Serial & Data & Value \\
\hline$(1)$ & Overall profit of the plant, $P_{t}$ & 500 \\
$(2)$ & Monetary penalty for downtime, $C_{U}$ & 100 \\
$(3)$ & Operation time, $T_{M}$ & 1000 \\
\hline
\end{tabular}

\section{Simulated Results}

6.1. Description of the Framework. The framework taken into account consists of $N_{n}=3$ nodes. All these nodes are in series. Every single node is made up of $N_{p}\left(i_{n}\right)=$ $3, i_{n}=1,2,3$, branches of single components in parallel $\left(N_{s}\left(i_{n}, i_{p}\right)=1 ; i_{n}, i_{p}=1,2,3\right)$. Components that belong to the similar node are supposed to be of the same type (thus, $N_{t}=N_{n}$ ) and therefore will be subjected to the identical maintenance strategy. Pictorial description of the abovementioned framework is shown in Figure 5.

Data that has been used for optimization of the said framework is tabulated in Table 1.

6.2. Case Study. In this case study, multiobjective genetic algorithm has been used for optimizing the system mean accessibility, “ $\bar{A}$," and the profitability, " $G$," over a given time " $T_{M}$." During this process, following assumptions are made with regard to the failure probabilities of the components in the framework that are resulting into different deterioration stages: 
TABLE 2: Parameters set for GA.

\begin{tabular}{llc}
\hline Serial & Data & Value \\
\hline$(1)$ & Number of chromosomes (population size) & 200 \\
$(2)$ & Number of generations (stopping criteria) & 200 \\
$(3)$ & Selection procedure & Roulette wheel \\
$(4)$ & Replacement strategy & Children-parents \\
$(5)$ & Probability of mutation & $1 \%$ \\
$(6)$ & Probability of crossover & $100 \%$ \\
$(7)$ & Generations considered without elitist selection & 100 \\
$(8)$ & Parents fraction picked with elitist selection & $25 \%$ \\
\hline
\end{tabular}

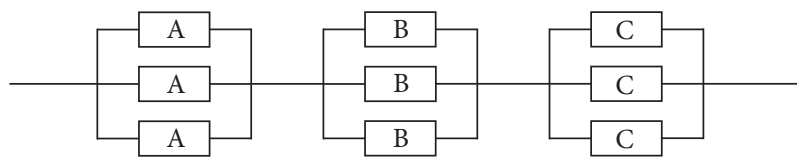

Figure 5: Pictorial description of framework considered in Section 6.1.

(i) The probabilities are not dependent on the load shared by the components.

(ii) The number of maintenance professionals is enough to maintain the faulty components of the framework simultaneously.

These conditions facilitate the application of analytical modeling as well as the validation of suggested coupled approach (GA + MC) on the described framework which is essential for their comparison. The design variables for optimization, that is, the thresholds, " $k_{\mathrm{th}}^{l}$ ", after which the preventive maintenance should be carried out for all component types, that is, $l=1,2,3$, are assumed to be selected within the array $(1,64)$. Parameters and rules set for the multiobjective genetic algorithm technique are mentioned in Table 2.

According to the assumptions, the search space, required to be optimized, consisted of a large number of alternatives, that is, $64^{3}$. The same were considered and the objective functions were assessed by utilizing Markov's model explained in Section 3. The results obtained after this analytical modeling of the framework are shown in Figure 6 (for the purpose of clarity, only corresponding objective functions' values with the multiples of 6 are shown). The processing time required for the said simulation on a Dell Inspiron N5110 Core-i5 was approximately 3 hours.

The results obtained by adopting the coupled approach $(\mathrm{GA}+\mathrm{MC})$ are depicted in Figure 7. Processing time elapsed for the procedure on the same machine was approximately $1.5 \mathrm{~min}$. The objective functions' values corresponding to the nondominated solutions are illustrated in this figure.

It can be clearly seen that the optimal solutions lie on the boundary of Pareto domination front. The same can be observed by making a comparison of Figures 6 and 7 . The difference of processing times taken by the analytical modeling as compared to the suggested approach highlights the effectiveness of the later for taking the search towards

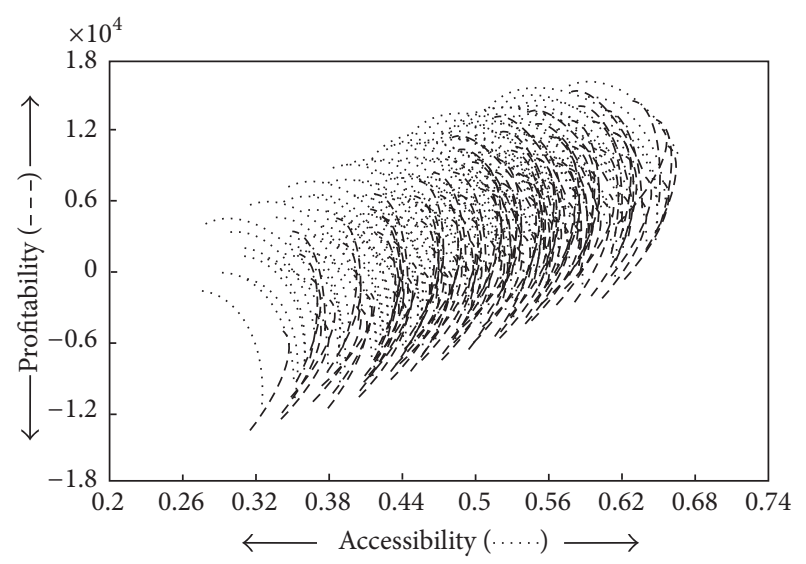

FIGURE 6: Graphical representation of objective functions' values evaluated by Markov's model.

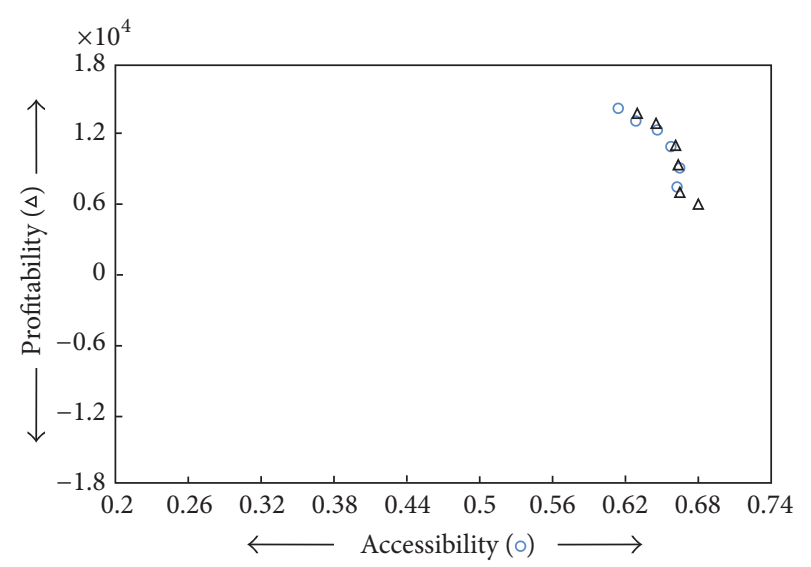

FIGURE 7: Graphical representation of objective functions' values evaluated by coupled approach.

the area of interest within the search space more rapidly. Objective functions' values for the nondominated solutions achieved as a result of adopting the suggested coupled approach are enumerated in Table 3.

\section{Conclusion}

The recognition of effective strategies, for repair/maintenance of plants/engineering systems, is of significant importance 
TABLE 3: Numerical values of objective functions for nondominated solutions.

\begin{tabular}{lcc}
\hline Serial & Accessibility, $\bar{A}$ & Profitability, $G$ \\
\hline$(1)$ & 0.6206 & $1.4 \times 10^{4}$ \\
$(2)$ & 0.6385 & $1.3 \times 10^{4}$ \\
$(3)$ & 0.6426 & $1.2 \times 10^{4}$ \\
$(4)$ & 0.6545 & $1.0 \times 10^{4}$ \\
$(5)$ & 0.6613 & $0.7 \times 10^{4}$ \\
$(6)$ & 0.6705 & $0.6 \times 10^{4}$ \\
\hline
\end{tabular}

from financial as well as safety point of view. Particularly, the benefits of carrying out condition-based maintenance have become more critical nowadays. Actually, the related phenomenon behind deterioration processes is very complex, particularly, when higher-risk frameworks, for instance, atomic power plants and offshore establishments, are concerned. In such cases, comprehensive simulation of the plant performance is very much essential, which can only be carried out analytically under simplified hypothesis.

This research framework is focused on the issue of finding an optimal condition-based maintenance strategy based on threshold levels of components deterioration beyond which maintenance is necessitated.

In order to deal with the issue, the problem is configured as a multiple objective search, to be dealt by a synergic approach, encompassing both GA and MC framework. The optimized level of deterioration for each element is explored while keeping in view the mean accessibility and net profit of the system. A population of chromosomes is considered by GA, each encoding an optimum level of deterioration for every specified element. For any particular chromosome, MC simulation approximates the two desired objective functions.

For validation of the proposed framework, in-built algorithms available in MATLAB for Multiple Objective Genetic Algorithms (MOGA) and MC simulation have been used. The approach has been implemented on a simple system. The obtained results have been compared with the ones acquired through analytical modeling of the system. These results show remarkable time saving with the proposed algorithm. In the next phase, we will consider the problems associated with stress-dependent deterioration processes of the system components as well as the limited quantity of maintenance professionals present on site. Keeping in view the achieved results, the proposed model has proved to be successful in contributing towards computational resources for searching the optimal solutions.

\section{Competing Interests}

The authors declare that they have no competing interests.

\section{References}

[1] P. K. Samanta, W. E. Vesely, F. Hsu, and M. Subudly, "Degradation modeling with application to ageing and maintenance effectiveness evaluations," Division of Systems Research, Office of Nuclear Regulatory Research, US Nuclear Regulatory Commission, 1991.

[2] J. A. M. Hontelez, H. H. Burger, and D. J. D. Wijnmalen, "Optimum condition-based maintenance policies for deteriorating systems with partial information," Reliability Engineering \& System Safety, vol. 51, no. 3, pp. 267-274, 1996.

[3] C. T. Lam and R. H. Yeh, "Optimal maintenance-policies for deteriorating systems under various maintenance strategies," Microelectronics Reliability, vol. 3, no. 36, p. 446, 1996.

[4] R. H. Yeh, "State-age-dependent maintenance policies for deteriorating systems with Erlang sojourn time distributions," Reliability Engineering \& System Safety, vol. 58, no. 1, pp. 55-60, 1997.

[5] A. Grall, C. Bérenguer, and C. Chu, "Optimal dynamic inspection/replacement planning in condition-based maintenance for deteriorating systems," in Proceedings of the European Conference on Safety and Reliability (ESREL '98), pp. 16-19, Trondheim, Norway, June 1998.

[6] V. A. Kopnov, "Optimal degradation processes control by twolevel policies," Reliability Engineering \& System Safety, vol. 66, no. 1, pp. 1-11, 1999.

[7] C. Bérenguer, A. Grall, and B. Castanier, "Simulation and evaluation of condition-based maintenance policies for multicomponent continuous-state deteriorating systems," in Proceedings of the Foresight and Precaution Conference, pp. 275-282, Edinburgh, UK, 2000.

[8] J. Barata, C. Guedes Soares, M. Marseguerra, and E. Zio, "Monte Carlo simulation of deteriorating systems," in Proceedings of the European Conference on Safety and Reliability (ESREL '01), pp. 879-886, Torino, Italy, September 2001.

[9] J. Barata, C. G. Soares, M. Marseguerra, and E. Zio, "Simulation modelling of repairable multi-component deteriorating systems for 'on condition' maintenance optimisation," Reliability Engineering \& System Safety, vol. 76, no. 3, pp. 255-264, 2002.

[10] Y. Sawaragi, H. Nakayama, and T. Tanino, Theory of MultiObjective Optimization, vol. 176, Elsevier, 1985.

[11] E. Borgonovo, M. Marseguerra, and E. Zio, "A Monte Carlo methodological approach to plant availability modeling with maintenance, aging and obsolescence," Reliability Engineering \& System Safety, vol. 67, no. 1, pp. 61-73, 2000.

[12] M. Cantoni, M. Marseguerra, and E. Zio, "Genetic algorithms and Monte Carlo simulation for optimal plant design," Reliability Engineering and System Safety, vol. 68, no. 1, pp. 29-38, 2000.

[13] S. Martorell, S. Carlos, A. Sánchez, and V. Serradell, "Constrained optimization of test intervals using a steady-state genetic algorithm," Reliability Engineering and System Safety, vol. 67, no. 3, pp. 215-232, 2000.

[14] J. H. Holland, Adaptation in Natural and Artificial Systems: An Introductory Analysis with Applications to Biology, Control, and Artificial Intelligence, University of Michigan Press, Ann Arbor, Mich, USA, 1975.

[15] J. H. Holland and D. E. Goldberg, Genetic Algorithms in Search, Optimization and Machine Learning, Addison-Wesley Longman, Boston, Mass, USA, 1989.

[16] D. Beasley, R. R. Martin, and D. R. Bull, "An overview of genetic algorithms: part 1. Fundamentals," University Computing, vol. 15, pp. 58-69, 1993.

[17] D. Beasley, D. R. Bull, and R. R. Martin, "An overview of genetic algorithms: Part 2, research topics," University Computing, vol. 15, no. 4, pp. 170-181, 1993. 
[18] C. M. Fonseca and P. J. Fleming, "Genetic algorithms for multiobjective optimization: Formulation, discussion and generalization," in Proceedings of the 5th International Conference on Genetic Algorithms, vol. 93, pp. 416-423, Morgan Kaufmann, San Mateo, Calif, USA, June 1993.

[19] D. B. Fogel, "An introduction to simulated evolutionary optimization," IEEE Transactions on Neural Networks, vol. 5, no. 1, pp. 3-14, 1994.

[20] D. W. Coit and A. E. Smith, "Use of a genetic algorithm to optimize a combinatorial reliability design problem," in Proceedings of the 3rd IIE Research Conference, pp. 467-472, Atlanta, Ga, USA, May 1994.

[21] N. Srinvas and K. Deb, "Multi-objective function optimization using non-dominated sorting genetic algorithms," Evolutionary Computation, vol. 2, no. 3, pp. 221-248, 1994.

[22] C. M. Fonseca and P. J. Fleming, "An overview of evolutionary algorithms in multi- objective optimization," Evolutionary Computation, vol. 3, no. 1, pp. 1-16, 1995.

[23] F. Herrera and M. Lozano, "Adaptation of genetic algorithm parameters based on fuzzy logic controllers," Genetic Algorithms and Soft Computing, vol. 8, pp. 95-125, 1996.

[24] G. T. Parks, "Muitiobjective pressurized water reactor reload core design by nondominated genetic algorithm search," Nuclear Science and Engineering, vol. 124, no. 1, pp. 178-187, 1996.

[25] P. A. Joyce, T. A. Withers, and P. J. Hickling, "Application of genetic algorithms to optimum offshore plant design," in Safety and Reliability, vol. 18, no. 3, pp. 6-12, Taylor \& Francis, Oxfordshire, UK, 1998.

[26] E. Zitzler and L. Thiele, "Multiobjective evolutionary algorithms: a comparative case study and the strength Pareto approach," IEEE Transactions on Evolutionary Computation, vol. 3, no. 4, pp. 257-271, 1999.

[27] E. Zitzler, Evolutionary Algorithms for Multi-Objective Optimization: Methods and Applications, 1999.

[28] V. G. Toshinsky, H. Sekimoto, and G. I. Toshinsky, "A method to improve multiobjective genetic algorithm optimization of a selffuel-providing LMFBR by niche induction among nondominated solutions," Annals of Nuclear Energy, vol. 27, no. 5, pp. 397-410, 2000.

[29] P. G. Busacca, M. Marseguerra, and E. Zio, "Multiobjective optimization by genetic algorithms: application to safety systems," Reliability Engineering \& System Safety, vol. 72, no. 1, pp. 59-74, 2001.

[30] M. Marseguerra and E. Zio, "Nonlinear Monte Carlo reliability analysis with biasing towards top event," Reliability Engineering \& System Safety, vol. 40, no. 1, pp. 31-42, 1993.

[31] M. Marseguerra and E. Zio, "Optimizing maintenance and repair policies via a combination of genetic algorithms and Monte Carlo simulation," Reliability Engineering \& System Safety, vol. 68, no. 1, pp. 69-83, 2000.

[32] A. Dubi, Monte Carlo Applications in Systems Engineering, John Wiley \& Sons, 2000.

[33] M. Marseguerra, E. Zio, and L. Podofillini, "Multiobjective spare part allocation by means of genetic algorithms and Monte Carlo simulation," Reliability Engineering \& System Safety, vol. 87, no. 3, pp. 325-335, 2005.

[34] Z. Feng, Markov process applied to degradation modelling: different modelling alternatives and their properties [M.S. thesis], Norwegian University of Science and Technology, 2013.

[35] L. Gacôgne, "Methods to apply operators in a steady-state evolutionary algorithm," in Proceedings of the IPMU Conference, Paris, France, July 2006. 


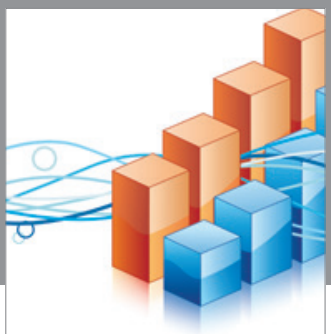

Advances in

Operations Research

vatem alat4

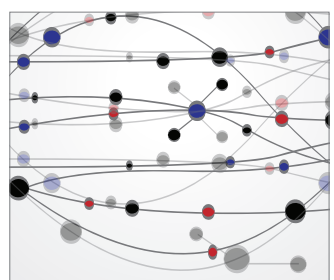

\section{The Scientific} World Journal
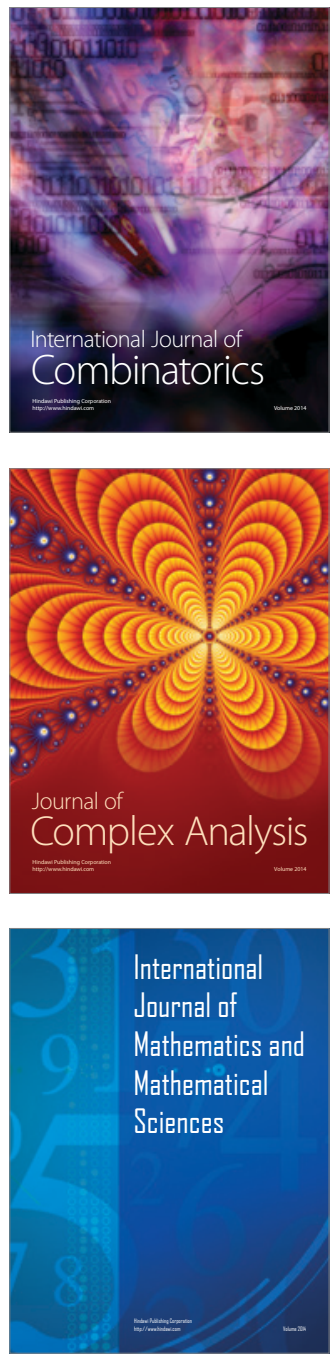
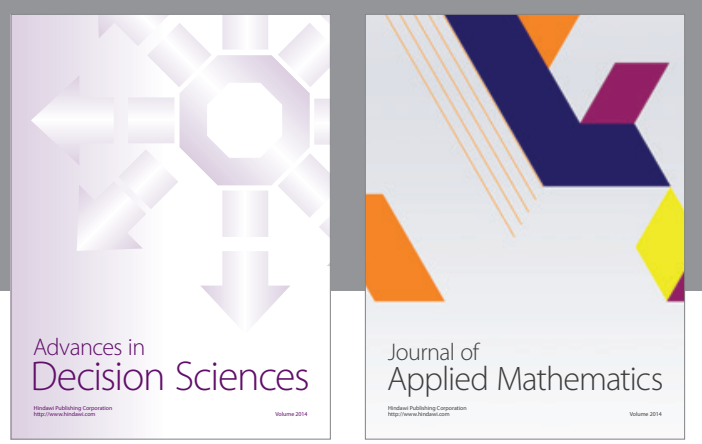

Algebra

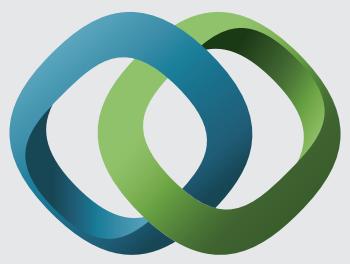

\section{Hindawi}

Submit your manuscripts at

https://www.hindawi.com
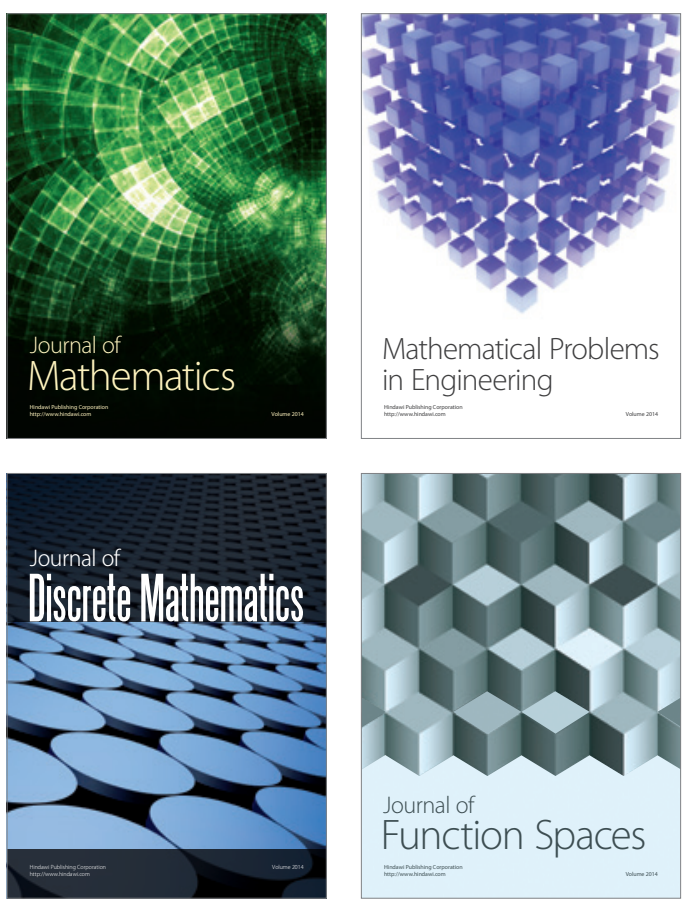

Mathematical Problems in Engineering
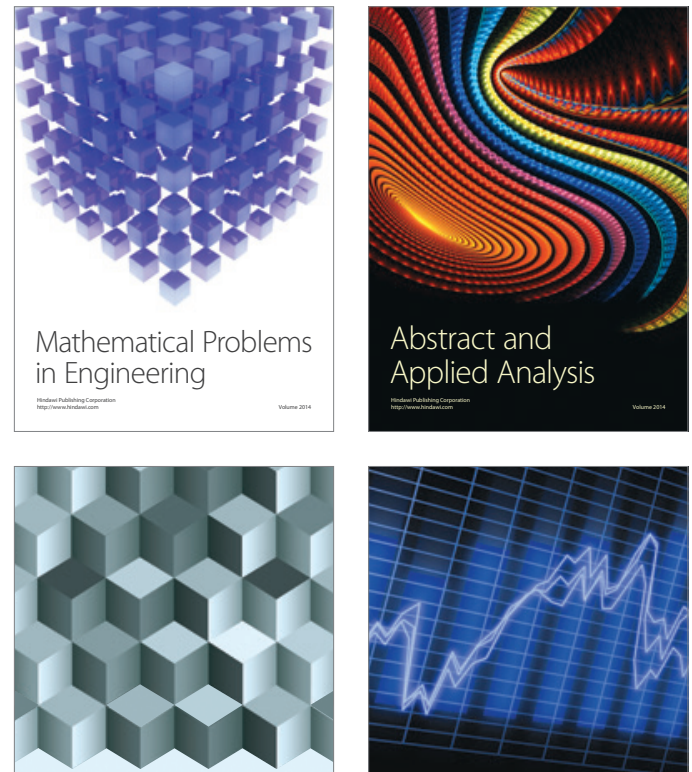

Journal of

Function Spaces

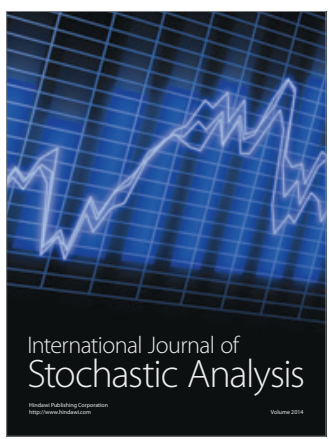

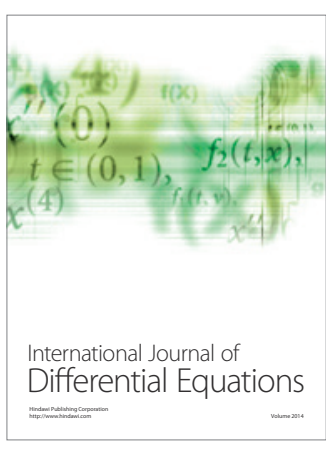
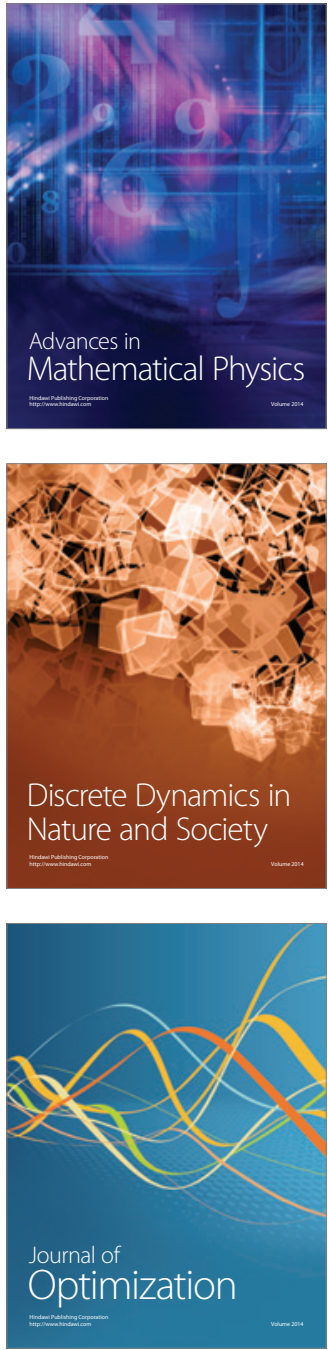